\title{
Forward Proton Detector on DØ Experiment
}

\author{
Alberto Santoro \\ Laboratório de Física Experimental de Altas Energias \\ Centro Brasileiro de Pesquisas Físicas \\ Rua Dr. Xavier Sigaud,150 22290-180 Rio de Janeiro - RJ -Brazil \\ E-mail:santoro@lafex.cbpf.br
}

Received 7 January, 2000

\begin{abstract}
This paper describes the Forward Proton Detector (FPD) proposed to be implemented in the Dzero Detector, as a set of sub-detectors consisting of 18 Roman Pots. We call attention to the importance of the studies of the diffractive region of the strong interactions.
\end{abstract}

\section{Introduction}

Since the discovery of Hard Diffraction by UA8Collaboration [1], we have had a significant development of the Diffractive Physics from both the theoretical and experimental point of view. From the experimental side we have the results coming from colliders, like Hera(H1 and Zeus) [2] and Tevatron (CDF and $\mathrm{D} \varnothing$ ), and from theoretical side we have many papers or contributions to the development of this subject [3]. These results are very important for the studies of the nature of the Pomeron, confirming that QCD has to take the diffractive hard scattering into account. Diffractive region constitutes a good laboratory to investigate how we can build the two aspects, soft and hard, of the strong interaction. Pomeron appears in both sides.

All the above experimental results constitute the data sample available to be used for theoretical and phenomenological models allowing the comparison with theoretical predictions. However many of these results have to be redone due to the poor statistics. Some of the processes studied have to be directly observed also. Information about $\mathrm{t}$ and $\xi$ distributions is missing to complete some studies and confirm (or not) some theoretical results. This information has a strong impact on future projects of colliders.

We intend to summarize here the Forward Proton Detector (FPD) project $^{1}$ [4] as a subset of detectors to be introduced in the $\mathrm{D} \varnothing$ spectrometer. This project will change qualitatively our Diffractive Physics data. This project was also motivated by the number of possible physics topics to be studied using the FPD and the recent development of the subject.

In the following sections we describe the FPD at $\mathrm{D} \varnothing$ and the main motivation to introduce these Roman
Pots on Tevatron beam-line. We end with a conclusion and perspectives.

\section{What is the FPD and What is our Motivation to Design New Roman Pots}

The FPD is a set of 18 Roman Pots using scintillation fibers as detectors, connected to multi-anode photomultipliers. The position of the Roman Pots on the both sides of the D $\varnothing$ detector (proton and anti-proton side) will allow the direct observation of the double Pomeron exchange and consequently all physics associated to this topology of the diffractive spectra.

The future scenario of the Diffractive Physics will be produced by a short number of experiments starting on the beginning of the next millennium. This is shown in the table 1.

The experiments at RHIC, Tevatron and HERA will start taking data in the next run of these colliders in the beginning of the next millennium. We do not have a precise information about the decisions for LHC.

Here we summarize the FPD project at Tevatron, $\mathrm{D} \varnothing$, as an addition to the whole upgrade of the detector. Fig. 1 shows the main modifications of the D $\varnothing$ detector. We will have a new DØ detector. The interested reader can go to the reference [5] to get more information about the general changes.

Finally we show an example of an event display from Dzero Run I, with a suggestion of possible geometry for Run II, triggering the event with the Roman Pots. This example shows in Fig. 2 a hard diffractive candidate and a double Pomeron exchange [4], one of the topologies to be studied.

\footnotetext{
${ }^{1}$ After have published the project as a proposal following the reference [4], we develop many points and achieve a final solution as we describe in this talk
} 
Table 1. This table shows the next future experiments for Diffractive Physics. In the table $1 \mathrm{BNL}=$ Brookhaven National Laboratory, RHIC = Relativistic Heavy Ion Collider, CDF and D $\varnothing$ are the two detectors at Tevatron, DESY = Deutsches Elecktronen Synchroton, H1 and ZEUS are the two detectors at HERA, and CMS, ATLAS, ALICE, are the detectors of LHC $=$ Large Hadron Collider of CERN. $\left({ }^{*}\right) \mathrm{We}$ do not have information about plans for Diffractive Physics at LHC.

\begin{tabular}{lll}
\hline Lab. \& Detector & Beam & C.M. Energy \\
\hline BNL - RHIC & p x p & $50-500 \mathrm{GeV}$ \\
FERMILAB & p x $\bar{p}$ & $2 \mathrm{TeV}$ \\
CDF and D & & \\
DESY & $\gamma \mathrm{p}$ & (e) $30 \mathrm{GeV}$ \\
H1 and ZEUS & & $(\mathrm{p}) 800 \mathrm{GeV}$ \\
CERN - LHC $(*)$ & $\mathrm{p} \mathrm{x} \mathrm{p}$ & $14 \mathrm{TeV}$ \\
CMS, ATLAS, & & \\
ALICE,...) & & \\
\hline
\end{tabular}

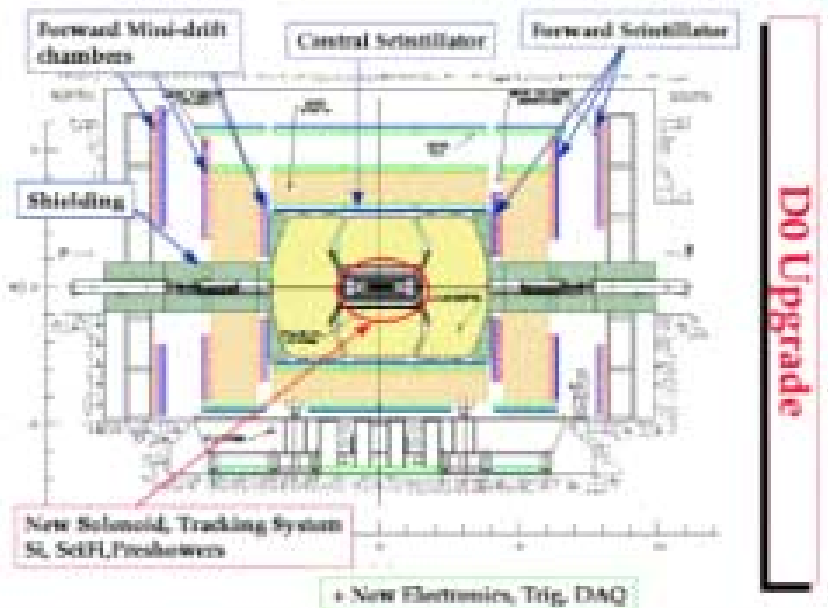

Figure 1. The D $\varnothing$ upgrade showing the main sectors being changed.

With the FPD we will be able to study an important number of new subjects and make better measurements that will allow us to address questions whose current data have left answered. These studies will be generally of two classes of events: those as a result of Soft Diffraction (SoD) and those produced by Hard Diffraction (HD). In SoD and HD we have topologies representing the Single Diffraction, Double Diffraction and the Double Pomeron exchange. We think that Soft Diffraction is well understood in the Regge phenomenology framework. Hard Diffraction is being studied in the present as well from the theoretical point of view as experimentally.

The only possible measurements missing on the side of the SoD will be done on colliders and FPD will be a strong tool to achieve this goal. The possible measurements to be done would be the total cross section and the $\mathrm{t}$-distributions $(\mathrm{t}=$ transfer momentum between the incoming proton (or anti-proton) and the scattered proton (or anti-proton)). We understand that it would be very important to have another measurement of the total cross section beyond those made by the CDF collaboration and by E811 at Tevatron [6]. These measurements have yet to be confirmed due to the consequences for the old and good phenomenology. The t-distributions would be an important help to get good models on the present.

Hard Diffraction is our main interest. We think that we can contribute to improve the understanding of many important subjects, like the hard Pomeron.

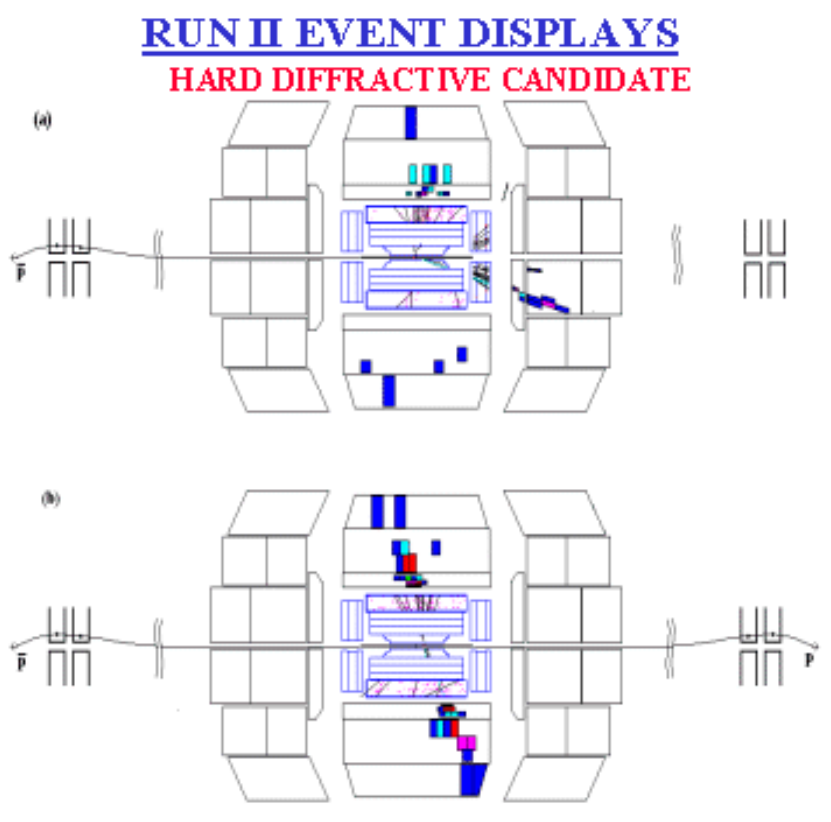

HARD DOUBLE POMERON CANDIDATE

Figure 2. The display of a possible double Pomeron exchange on Dzero detector.

\section{II.1 Some Topics of Physics to be ex- ploited by the FPD in the next Tevatron run}

Before going to the physics topics we would like to stress that the rapidity gap is an important tool to identify events associated to an specific topology. Rapidity gap is the interval of rapidity without particle production or without color activity. In order to get a diffractive event without Roman Pots we have almost always to do an offline analysis. We first plot the multiplicity events and count the number of events with multiplicity $\mathrm{n} \approx 0$. After that we build a lego plot with $\eta \times \phi$ variables, where,

$$
\eta=-\ln \left(\tan \frac{\theta}{2}\right)
$$

is the pseudo-rapidity, referred here sometimes simply as rapidity; $\theta$ and $\phi$ are the polar and azimuthal angles of the object produced diffractively. In the case of 
the hard diffraction, they are jets. We associate to each topology a lego plot. The Fig. 3 shows these topologies.

We observe that the lego plot corresponding to the elastic process does not present any particle in all spectrum of $\eta$. The produced particles are represented by the dots on the lego plot, while jets are represented by the small circles. The difference between hard and soft process is the presence of jets on the lego plot.
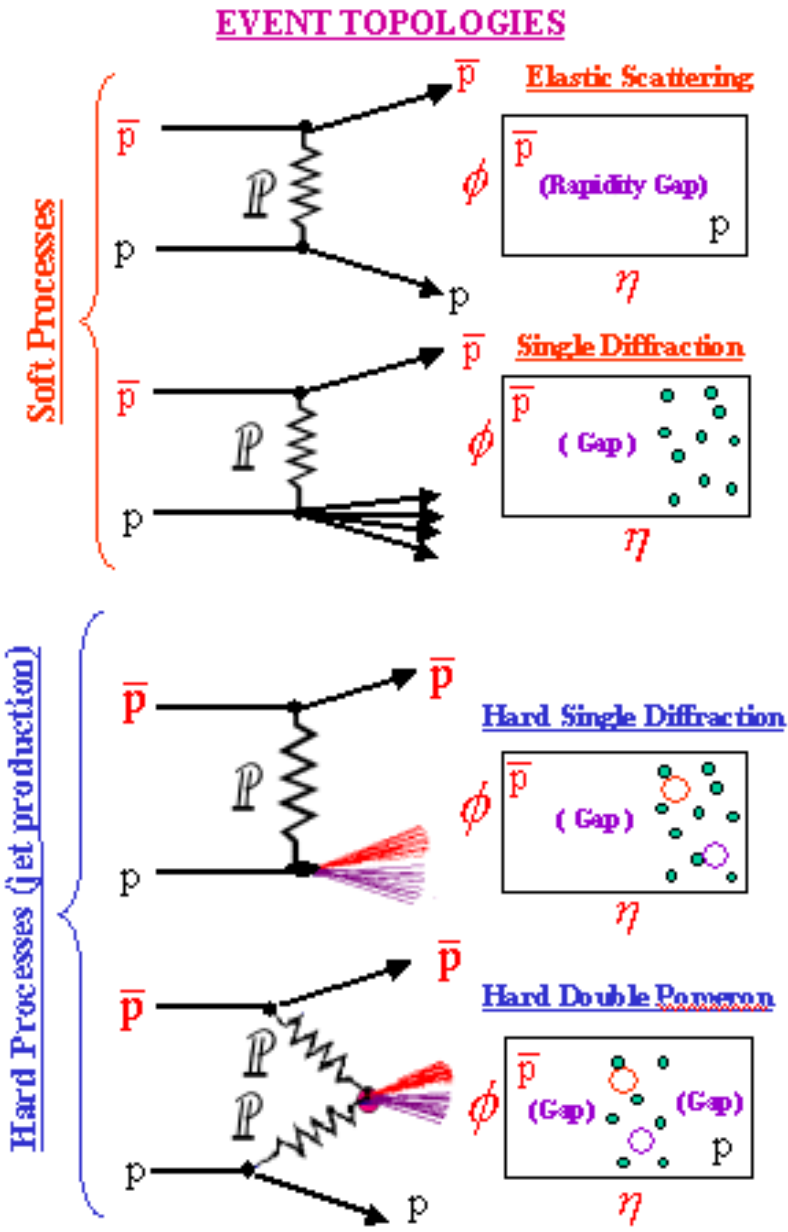

Figure 3. Some of the possible topologies and the associated lego plot. We see on the top the soft processes with the elastic and the single diffraction. On the down part of the figure we see the processes of hard single diffraction and the double Pomeron exchange.

\section{Diffractive Jet production}

Jets have been largely studied by QCD. The discovered of diffractively produced jets [1] by UA8 collaboration was very important for diffractive physics. It is expected that single and double jet diffractive production be exhaustively studied in the next future with FPD, making a distinction to those produced by the flux of color interactions.

\section{Low and High $|t|$ elastic scattering}

It still important to have new measurements of the Elastic scattering. Some of the present pro- posals for new experiments are to measure the elastic scattering for $\mathrm{pp}$ and $p \bar{p}$ in the region of low transfer momentum. With the FPD, D $\varnothing$ will be able to measure both, high and low t for elastic scattering. These measurements will be complementary to measurements from other experiments. This will allow to give a large number of points of t-distribution. In a particular way, the measurements of elastic cross sections give a direct connection to total cross section via the $\mathrm{Op}$ tical Theorem. It is important to know the elastic slope of the differential cross section. The value of the slope characterizes a specific process which can be associated to a particular production (e.g. resonances production).

\section{Total cross section}

We have new conflicting results from Tevatron [6] experiments.The measurement of the total cross section is very important to test the Froissart Bound which is derived only from the general principles of physics. An experiment is necessary to measure this cross section so we can know more accurately how the cross sections depends on the energy. After the Tevatron only the LHC will offer a new opportunity to make these measurements. The measurements made at the Tevatron will have a big impact on the future measurements at higher energies.

\section{Diffractive $\mathrm{W} / \mathrm{Z}$ boson production}

The present results for diffractively produced $\mathrm{W}$ and $\mathrm{Z}$ bosons are not satisfactory. It is important to understand these processes better to have a comparison with the current boson production. Both CDF [7] and DØ have made progress and the present results are motivating both collaborations to proceed new measurements.

\section{Diffractive Heavy Flavor Production}

Heavy Flavor physics, including the Diffractive production, has long been studied. We could separate the heavy flavor sector in three types of particles and corresponding physics.

i- The c-quark (charm), that although, not heavy enough is apparently copiously produced diffractively. [8] Charm physics is the threshold to heavy flavor physics.

ii- The b-quark (bottom) is heavy enough and it is in good agreement with QCD predictions.

iii- The t-quark (top) is very heavy, announcing perhaps a threshold to "new physics"

However, heavy flavor physics is considered almost as a high $p_{t}$ physics only. Diffractive heavy 
flavor production has not sufficiently being studied. This is due mainly to the absence of experimental results in this area for lack of adequate apparatus to observe Diffractive production of heavy flavor. One interesting ratio to be measured is studied in reference [8].

$$
\frac{\text { Diff.Heavy Quark }}{\text { All Diff. events }}>\frac{\text { Heavy Quark Events }}{\text { All Events }}
$$

\section{Inclusive single diffraction}

The inclusive single diffraction, has many subjects associated with it. Particularly for the Tevatron detectors, the Diffractive mass available, for single diffraction events, $M_{x}=450 \mathrm{GeV}$, makes the extraction of heavy flavor physics comfortable. Inclusive single diffraction has been a good laboratory for several problems in diffraction physics. We intend to use it to study jets and to calculate ratios between cross sections of different processes.

\section{Hard Double Pomeron Exchange}

An advantage of the large Diffractive mass produced at Tevatron, in this case $M_{x}=100 \mathrm{GeV}$, is the possibility to study by direct observation the Pomeron $\times$ Pomeron interactions and the associated physics. The instrumentation proposed by FPD/ DØ is appropriated to face the challenges of the double Pomeron mechanism to produce several objects not yet observed.

\section{Glueballs}

Since the origin of QCD, Glueballs have being studied by theoreticians and experimentalists. However, we do not have a significant progress in this subject. We need more experiments dedicated to the discover of glueballs without ambiguity with quark anti-quark competitive states. The family of glueballs is big. Table 2 shows the glueballs (oddballs are also shown). Oddballs should have the priority to be examined due to the fact that they do not have competition with natural q $\bar{q}$ states, mesons, and the qqq states, (baryons) with the same quantum numbers. It is difficulty to separate the common hadrons from the glueballs when they appear in the same physical region.

Glueballs are important for QCD that predicts their existence. Since Pomeron can be interpreted as glueballs, the study of hard diffraction in the QCD framework is an interesting subject to be developed.

\section{Centauros}

Centauros were have never been observed in accelerator particle physics. These objects were discovered in Cosmic Ray Physics as an event with several unusual characteristics, like the production of a large multiplicity of charged particles accompanied by very few photons. For example, as many as 100 charged particles and no more than $3 \pi^{0}$. [9] We have enough energy at Tevatron to produce centauros. Since our diffractive mass is significatively high we can produce it diffractively. The calorimetry of the detectors can be used to observe the absence of electromagnetic activity.

\section{Diffractive Structure Functions}

The study of Diffractive structure functions at Tevatron would allow a comparison with the existing Hera results. To understand the structure of the Pomeron one must know its structure function. This type of study has to be pursued exhaustively to get better accuracy and to be possible a clear interpretation Pomeron. How important is the gluon and the quark component of the Pomeron. With this results we can have better calculations of its cross sections[10]. Is the Pomeron the same in electron proton and proton anti-proton interactions? There are difference between the Pomeron structure in different reactions?

Table 2. This table shows some of the possible quantum number configurations for glueballs. The Oddballs are the glueballs with quantum number without a competition with known hadrons.

\begin{tabular}{||c|c|c|c|c||}
\hline \hline$J^{P C}$ & $(q \bar{q})$ & $2 \mathrm{~g}$ & $3 \mathrm{~g}$ & ODDBALLS \\
\hline \hline $0^{++}$ & Yes & Yes & Yes & No \\
\hline $0^{+-}$ & No & No & Yes & Yes \\
\hline $0^{-+}$ & Yes & Yes & Yes & No \\
\hline $0^{--}$ & No & No & Yes & Yes \\
\hline $1^{++}$ & Yes & Yes & Yes & No \\
\hline $1^{+-}$ & Yes & No & Yes & No \\
\hline $1^{-+}$ & No & Yes & Yes & Yes \\
\hline $1^{--}$ & Yes & No & Yes & No \\
\hline $2^{++}$ & Yes & Yes & Yes & No \\
\hline $2^{+-}$ & No & No & Yes & Yes \\
\hline $2^{-+}$ & Yes & Yes & Yes & No \\
\hline $2^{--}$ & Yes & No & Yes & No \\
\hline $3^{++}$ & Yes & Yes & Yes & No \\
\hline $3^{+-}$ & Yes & No & Yes & No \\
\hline $3^{-+}$ & No & yes & Yes & Yes \\
\hline $3^{--}$ & Yes & No & Yes & No \\
\hline \hline
\end{tabular}

11. Correlations between $\eta, \mathbf{t}, M_{x}, \mathbf{b}, \xi, \mathbf{x}, E_{T}, \ldots$ It is important to study systematically the correlations between the kinematical variables $\eta$, $\mathrm{t}$, 
$M_{x}, \mathrm{~b}, \xi, \mathrm{x}, E_{T}, \ldots$ as well as to obtain the single distributions for each one of these variables. These studies are a phenomenological source of investigation of the hidden dynamics of the distributions. $\eta$ the pseudo-rapidity, (as defined above) is very useful to build the Lego Plots of $\eta \times \phi$ where $\phi$ is the azimuthal angle of the object being studied (e.g. jets); $t=\left(P_{\text {Beam }}-P_{\text {Scattered }}\right)^{2}$ is the transfer momentum between the proton beam and the scattered proton ; $M_{x}=\sqrt{\xi} \sqrt{s}$ is the diffractive mass ( 450 . GeV for single diffraction and 100. GeV for double Pomeron exchange in the energies of the Tevatron $(\sqrt{2} \mathrm{TeV}))$; $\mathrm{b}$ is the measured slope of the differential cross sections, which can be selected globally or for a particular region of the invariant mass produced diffractively $\left(\frac{d \sigma}{d t} \propto e^{-b\left(M_{x}\right) t}\right) ; \xi=1-x_{p}=\frac{\Delta P}{P}$ is the fraction of the momentum of the proton carried by the Pomeron; $x_{p} \geq 0.95$ is the fraction of the momentum of the proton carried by the scattered proton; and $E_{T}=$ is the transverse energy of the jet produced by hard diffraction.

With the FPD, we can get a good sample of Diffractive events and carry on these studies and others eventually suggested. This sample can be estimated and compared with the present experiments as is shown in table 3 .

Table 3. This table shows a comparison between what we have and what is estimated using FPD at the Run II of the Tevatron.

\begin{tabular}{||c|c|c||}
\hline \hline Experiment & Dijet Events & $E_{T}[\mathrm{GeV}]$ \\
\hline \hline UA8 & 100 & 8 \\
\hline HERA & Hundreds & 5 \\
\hline CDF & Thousands & 10 \\
\hline \multirow{3}{*}{ DØ /FPD } & 500,000 & 15 \\
\cline { 2 - 3 } & 150,000 & 20 \\
\cline { 2 - 3 } & 15,000 & 30 \\
\hline \hline
\end{tabular}

\section{The Forward Proton Detec- tor}

We will give just an idea about the FPD since we have already described the studies and project on reference [4].

\section{III.1 Generalities}

The Forward Proton Detector consists of 18 Roman Pots arranged on both sides of the DØ detector. Fig. 4 shows the Roman Pots on beam line. We have two castles on the proton side indicated by $P_{1 Q(S)}$. For analogy $P_{2 Q(S)}$ are the pots of the down position. On the anti-proton side we have two similar castles labeled by $A_{1 Q(S)}$ and two others half castles on the side of the dipole magnet labeled $A_{1(2) D}$. The approximated distances of the pots with respect to the interaction point (indicated by 0 on the ruler) are shown.

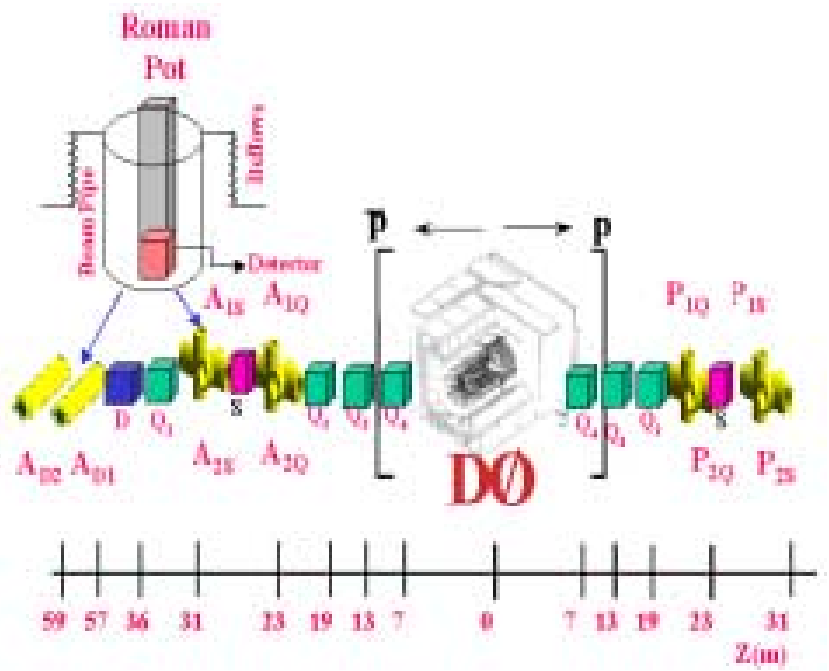

Figure 4. The Roman Pots position on the Tevatron beam line.

\section{III.2 Tevatron Reconfiguration}

We had to work on the beam line to open room for the FPD stations. We show in the Fig. 5 the real position of the Castles. We see that the cryogenic bypass is bigger in the Run II. This was the only place where we could open the necessary space for the FPD. Fig. 5 also shows that the quadrupole magnet $Q_{1}$ is no longer present. Other small modifications were necessary, like drilling a hole on the floor to allow the insertion and removal of the bottom detectors. Summarizing the Tevatron modification are:

- girder modification

- new cryogenic bypass

- removal of $Q_{1}$ quadrupole.

\section{III.3 Roman Pots}

In this subsection we will try to give a short description of Roman Pots's shown in the Fig. 6.

\footnotetext{
${ }^{2}$ To avoid some confusion about the names used for our detectors we use the standard notation for Roman Pots. Nevertheless, the more technically sophisticated Roman Pots, oblige us to note several parts which are being systematically cited in this text: a Castle which is shown in Fig. 6 in which we put the tube with the pot. Inside of the pot are the scintillating fibers constituting the detector. Some times we refer to the detectors to designate the final complex of parts and some times we use for the same thing the generic name Roman Pots.
} 
Tevatron Reconfliguration
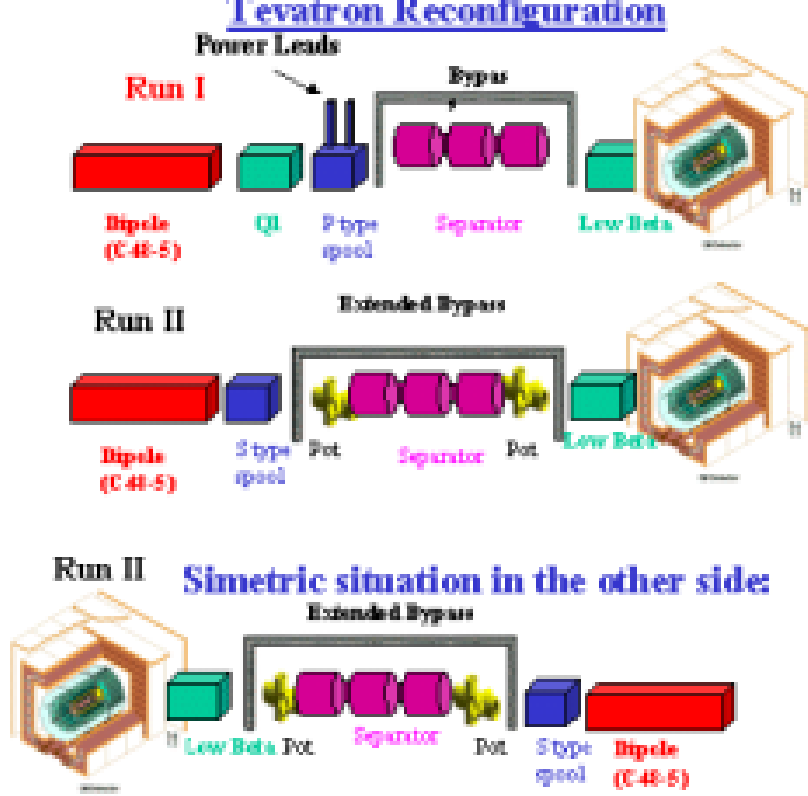

Figure 5. The Tevatron Reconfiguration or the Tevatron before and after the introduction of the Castles with the Roman Pots.

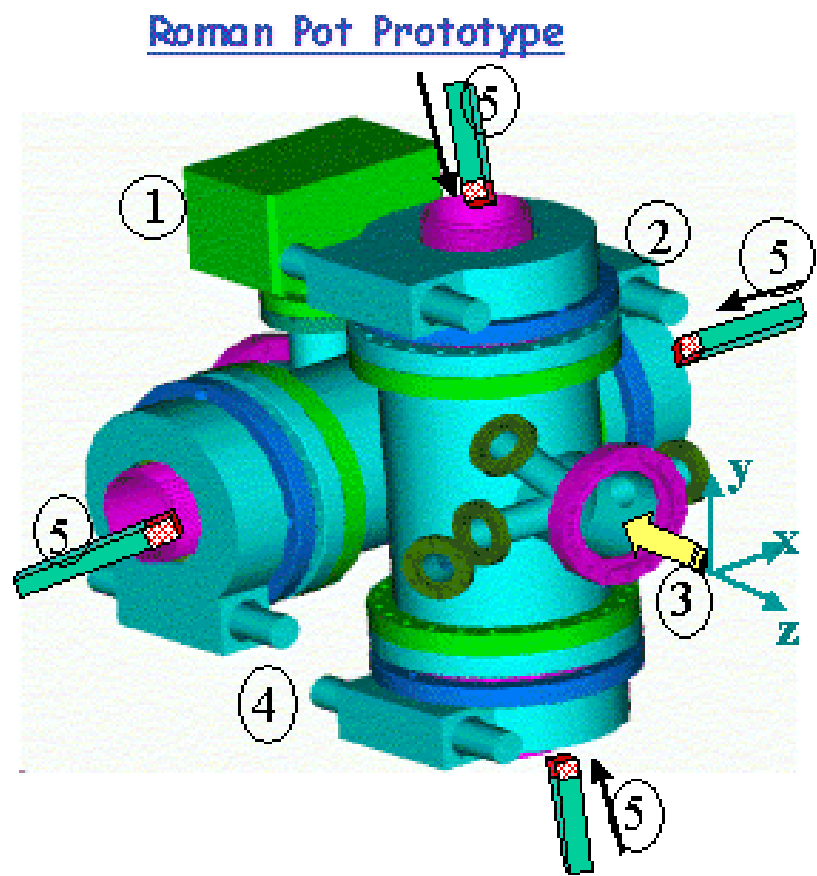

Figure 6. The Castle and the Roman Pots. The numbers, in this figure means, $1=$ ion pump; $2=$ worm gear; 3 $=$ Beam direction; $4=$ step motors; $5=$ the cartridge with the detectors inside.

Fig. 6 shows a FPD castle. The system of axes shown indicates the beam in the z-direction. The inner part of the castle will be in the UHV (ultra high vacuum) vacuum of the beam line. An ion pump shown as "1" in the figure is used to guarantee the UHV. Indicating by the number "2" we have a worm gear assembly that is the piece responsible for the moving mechanism of the pot itself. The beam direction is shown as number "3". The number "4" indicates the step motors used to move the pots. There are 4 step motors, one for each pot, all remotely operated. Finally we see the cartridge with the pot in its extremity indicated by the number " 5 ".

\section{III.4 The Detectors}

Fig. 7 shows our detector. The detector is constituted by scintillating fibers placed in the frames represented in the Fig. 7 by the planes X X', U U' and V V' . The scintillating fibers are connected to clear fibers which guide the signal up to the multi-anode photomultipliers as shown in the figure. We have 16 channels per plane X X' and 20 channels/plane U U', V V', giving a total of 112 channels per detector and 2016 channels total. Studies about the signal, efficiency and resolution have been made. Scintillating fibers are the best option for our detectors among many other possible technologies. The frame is made of ordinary plastic. The theoretical resolution is 80 microns.

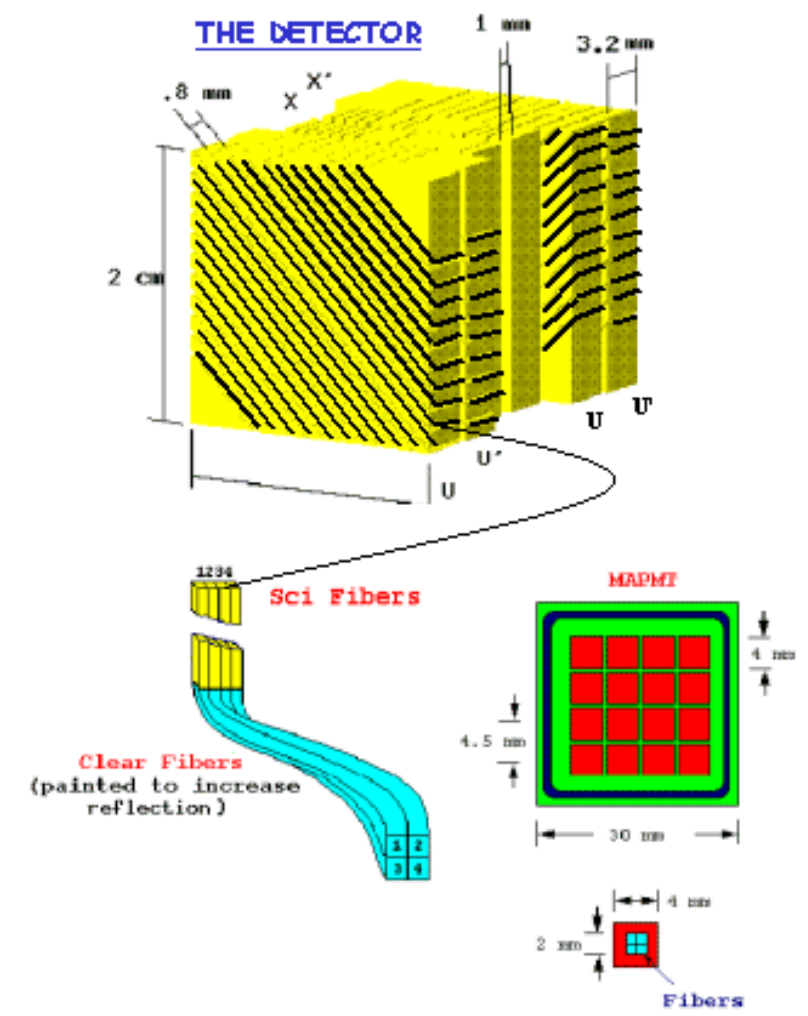

Figure 7. This figure shows our detectors and the multianode photomultipliers.

The acceptance of our detectors has been studied in several views. We show in Fig. 8 the acceptance versus the pot position. While the figure shows an elliptical with a distance of $8 \sigma$, the points in the plot are the result of the simulation for several distances. The line connecting the points is a guide eye only. 


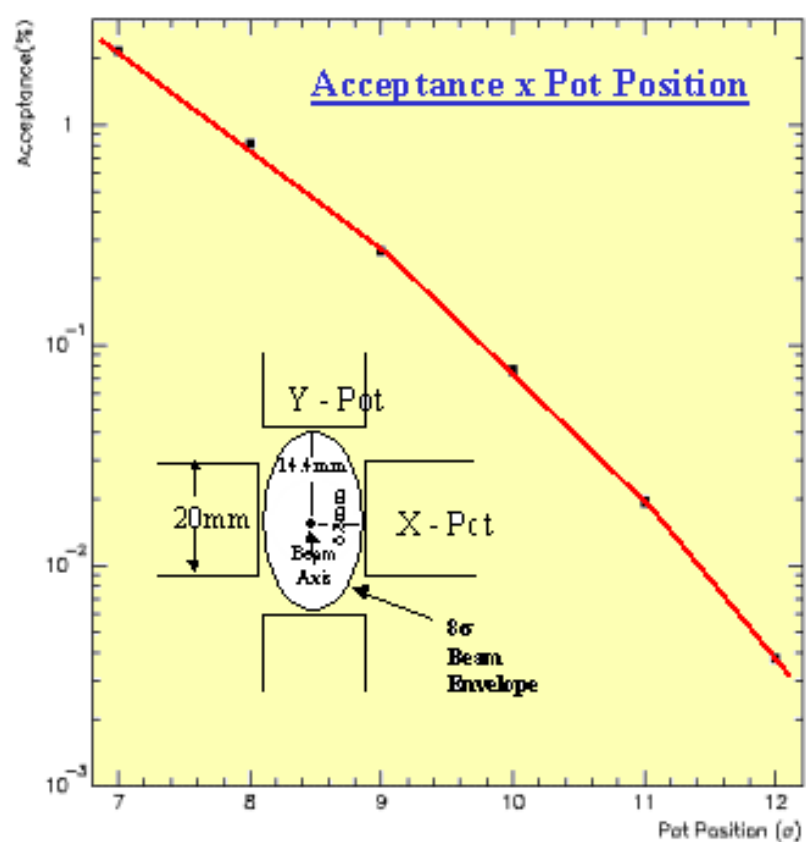

Figure 8 . This figure shows studies of the acceptance versus pot position.

\section{Conclusion}

The primary physics goal of the FPD is to measure hard diffraction, producing new data, turning the study new diffractive physics possible. On the other hand a number of measurements does not have enough statistics to get more accurated results. Nevertheless it will be possible to use the FPD to reduce uncertainties on the luminosity for all $\mathrm{D} \emptyset$ physics processes.

We can also obtain results and improve old measurements at lower energies and, in some cases, decide between conflicting results as is the case of the total cross sections. We have given, a list of possible topics to be investigated in both hard and soft diffraction.

Our schedule to start the data acquisition is the same of the Dzero Detector, i.e., by the end of the year 2000 .

This project will give the possibility to upgrade the world diffractive data since many new features will be possible, like the direct observation of the Pomeron Pomeron scattering.

Finally we would like to end this summary of this talk about the FPD project, with the photo of the prototype of our Roman Pot shown in Fig. 9

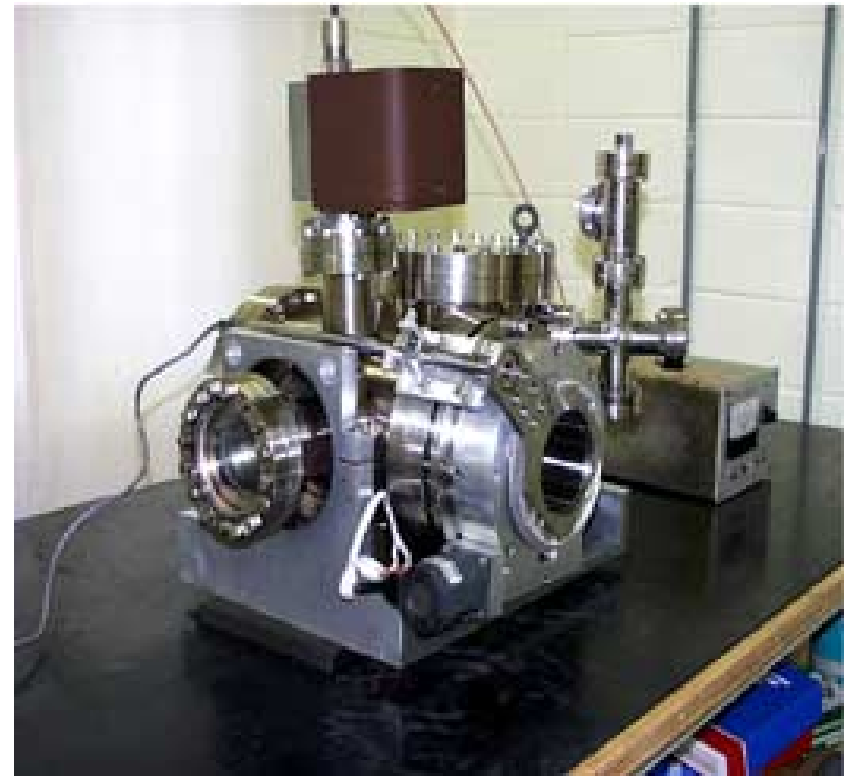

Figure 9. This is the final prototype of our Roman Pot or the Castle build at Laboratório Nacional de Luz Sinchroton, Campinas/Brazil.

I would like to thank the organizer committee of the XX-ENFPC for giving me the opportunity to present our FPD project. All the work behind this paper was done with the effort and contribution of the DØ collaboration in particular to A. Brandt and M. Martens from Fermilab. I would also like to thank our regional (Brazilian) collaboration made of colleagues from Universidade Federal da Bahia (N.Oliveira); Universidade Federal do Rio de Janeiro (J.Barreto); Universidade Estadual do Rio de Janeiro (W.Carvalho, C. Martins, V. Oguri and A. Sznajder); Universidade Estadual Paulista (E. Gregores, T. Lungov and Sergio Novaes); and Laboratorio Nacional de Luz Synchroton (R.Neuezwander and M. Juni). Without the important contribution and cooperation of Ricardo Rodrigues and Cylon Goncalves from LNLS we could not have reached this point in this project. Finally I would like to thank my colleagues of laboratory, (LAFEX/CBPF) G. Alves, M. Miranda, H. da Motta, M. Souza and M. Vaz for all the work that they are doing in this project. We thank $\mathrm{CNPq}$ and FAPERJ for partial financial support.

\section{References}

[1] A.Brandt et al., UA8 Collaboration, Nucl. Inst. Meth. A 327, 412 (1993); id. , Phys. Lett. B 297, 93 (1993); ibid. , HEP-Ex-9709015, 1997, submitted to PLB

[2] ZEUS collaboration, M. Derrick et.al., Z. Phys. C 65, 379(1995); H1 collaboration, T. Ahmed et.al., Nucl. Phys. B 439, 471 (1995); ZEUS collaboration, M. Derrick et.al. Z. Phys. C 68, 569 (1995); H1 collaboration, T. Ahmed et.al. Phys. Lett. B 348, 681 (1995). 
[3] A. Brandt, H. Motta and A. Santoro -EditorsLISHEP98- Lafex International School on High Energy Physics - Proceedings of Session C -February 1620,(1998)

[4] A. Brandt et al. A Forward Proton Detector FermilabPub-97/377, 1997

[5] DØ Collaboration, Fermilab Pub-96/357-E, (1996).

[6] C.Avila, Proceedings of LISHEP98; Thesis: "Measurement the proton - antiproton total cross section at center of mass Energy of $1800 \mathrm{GeV}$ " Cornell University 1997 and references therein; C.Avilez et al., Phys. Lett. B 234, 158 (1990);ibid. , Phys. Rev. Lett. 68, 2433 (1992); ibid., Phys. Rev. 50, 5550, (1994); ibid. , Phys. Lett. B 234, 158 (1990); ibid. , Nucl. Inst. Meth. A 360, 80 (1995)
[7] F.Abe et al. CDF Collaboration: Phys. Rev. Lett. 78, 2698 (1997)

[8] E.L.Berger, J.C.Collins, D.E. Soper, G.Sterman, Nucl. Phys. B 286, 704 (1987); A. Kerman and G.Van Dalen, Phys. Rep. 106, 297 (1984)

[9] F. Halzen: Felix Home Page, Brazil-Japan Collaboration - Proceedings of the 21st. Int.Conf.-Adelaide-Australia vol. 8, 259 (1990); C. M. G. Lattes, Y. Fujimoto and S. Hasegawa, Phys. Rep. 65, 151 1980; C. E. Navia et al.: Phys. Rev. D 40, 2898 (1989).

[10] R. J. M. Covolan and M.S. Soares, IFGW-DRCC 97/04(1997) "A Study on the Pomeron Structure Function". 